\title{
The Current Epidemic of the Barley Pathogen Ramularia collo-cygni Derives from a Population Expansion and Shows Global Admixture
}

\author{
Remco Stam, ${ }^{1, \dagger}$ Hind Sghyer, ${ }^{1}$ Aurélien Tellier, ${ }^{2}$ Michael Hess, ${ }^{1}$ and Ralph Hückelhoven ${ }^{1}$ \\ ${ }^{1}$ Chair of Phytopathology, School of Life Sciences Weihenstephan, Technical University of Munich, 85354 Freising, Germany \\ ${ }^{2}$ Section of Population Genetics, School of Life Sciences Weihenstephan, Technical University of Munich, 85354 Freising, Germany \\ Accepted for publication 17 July 2019.
}

\begin{abstract}
Ramularia leaf spot is becoming an ever-increasing problem in main barley-growing regions since the 1980 s, causing up to $70 \%$ yield loss in extreme cases. Yet, the causal agent Ramularia collo-cygni remains poorly studied. The diversity of the pathogen in the field thus far remains unknown. Furthermore, it is unknown to what extent the pathogen has a sexual reproductive cycle. The teleomorph of $R$. collo-cygni has not been observed. To study the genetic diversity of $R$. collo-cygni and get more insights in its biology, we sequenced the genomes of $19 R$. collo-cygni isolates from multiple geographic locations and diverse hosts. Nucleotide polymorphism analyses of all isolates shows that $R$. collo-cygni is genetically diverse worldwide, with little geographic or host specific differentiation. Next, we used two different methods to detect signals of recombination in our sample set. Both methods find putative recombination events, which
\end{abstract}

ABSTRACT

indicate that sexual reproduction happens or has happened in the global $R$. collo-cygni population. Lastly, we used these data on recombination to perform historic population size analyses. These suggest that the effective population size of $R$. collo-cygni decreased during the domestication of barley and subsequently grew with the rise of agriculture. Our findings deepen our understanding of $R$. collo-cygni biology and can help us to understand the current epidemic. We discuss how our findings support possible global spread through seed transfer, and we highlight how recombination, clonal spreading, and lack of host specificity could amplify global epidemics of this increasingly important disease and suggest specific approaches to combat the pathogen.

Keywords: ecology and epidemiology, population biology
The Dothidiomycete Ramularia collo-cygni was originally described as a parasitic agent on grasses as early as 1893 (Cavara 1893). Yet, it was only in the mid-1980s that it was rediscovered as the causal agent of the Ramularia leaf spot (RLS) disease of barley, and it is recognized as an important pathogen for barley worldwide (Havis et al. 2015). The development of molecular diagnostics has confirmed the unsymptomatic presence of the pathogen throughout the lifecycle of its host barley (Havis et al. 2015). The pathogen has become increasingly problematic in many of the world's barley-growing regions, affecting the yield often by up to $25 \%$ because of reduced kernel size and limited growth. In extreme cases, up to $70 \%$ yield reduction has been observed. These problems are intensified by the fact that $R$. collo-cygni resistance in existing barley cultivars is often of quantitative nature and remains limited. In fact, certain cultivars already seem to have lost resistance against the pathogen. Additionally, $R$. collo-cygni shows increasing fungicide resistance (Havis et al. 2015).

The reasons for the emergence of RLS remain largely unknown. The current epidemic of RLS has followed the intensive use of mildew-resistant mlo genotype cultivars. McGrann et al. (2014) suggested a tradeoff between the barley mlo mutation-mediated

†Corresponding author: R. Stam; stam@wzw.tum.de

Funding: This work was, in part, financially supported by Bavarian State Ministry of Food, Agriculture and Forestry projects KL/08/07 (to M. Hess and R. Hückelhoven) and KL/12/07 (to M. Hess and R. Hückelhoven) and the Bavarian State Ministry of the Environment and Consumer Protection in frame of project network BayKlimaFit (to R. Hückelhoven; subproject 10).

A. Tellier, M. Hess, and R. Hückelhoven contributed equally to this work.

*The $e$-Xtra logo stands for "electronic extra" and indicates that six supplementary figures are published online.

The author(s) declare no conflict of interest.

(C) 2019 The American Phytopathological Society powdery mildew resistance and susceptibility to RLS. However, the disease is not restricted to mlo genotypes, and analysis of nearisogenic $M L O$ and $m l o$ barley did not support a causal link between the mlo mutation and the amount of $R$. collo-cygni DNA detected in barley seeds (Hofer et al. 2015). Additional physiological properties of modern barley varieties, climate change, and an extended growing season might have increased the host susceptibility (West et al. 2012).

The first draft genome of the pathogen was published in 2016 and showed a low number of cell wall-degrading enzymes (CWDEs) as well as a large amount of secondary metabolite clusters (McGrann et al. 2016). These findings could partly be explained by the lifestyle of $R$. collo-cygni, because the fungus could have evolved these as a strategy to avoid detection of cell wall elicitors by the plant during the relatively long asymptomatic growth. However, large-scale comparative analysis of CWDEs in different pathogenic and nonpathogenic fungi revealed overall large variation and no correlation to pathogenicity or host (Zhao et al. 2013).

We generated an independent draft genome for another isolate (urug2) with improved scaffold length and showed that $R$. collocygni strongly diverged from Zymoseptoria tritici, its nearest sequenced sister species, about 27 million years ago (Stam et al. 2018). The $R$. collo-cygni genome does not show many of the typical plant pathogen characteristics, like transposable element islands or elevated rate of nonsynonymous over synonymous substitutions (dN/dS) in secreted or effector proteins, which would indicate selective pressure on such genes. We hypothesized that these genome characteristics are linked to the largely endophytic lifestyle of the pathogen. Yet again, they do not explain its emergence as an epidemic threat.

In addition to the lack of basic genomic knowledge, questions also remain regarding the transmission of the fungus itself. Some authors hypothesize that, based on the regular detection of $R$. collocygni from grain and microscopy studies of seedlings, seed 
transmission plays an important role in the current epidemic (Havis et al. 2014; Kaczmarek et al. 2017). Others have largely attributed the spread of epidemics to wind dispersal (Schützendübel et al. 2008). It has also been suggested that seed transmission would facilitate long-distance movement, whereas wind dispersal is responsible for local spread (Havis et al. 2015).

Other than being found on barley, $R$. collo-cygni has also been isolated from wheat, oat, maize, and a number of uncultivated grasses, such as Agropyron repens, suggesting a broad host range (Salamati and Reitan 2006). The compatible interactions with many of these grasses are microscopically similar to the infection of barley (Kaczmarek et al. 2017). Despite a similar initial stage, the reaction on dicotyledonous plants is found to be incompatible, which shows some degree of host specialization for grasses. It is so far not known whether isolates of $R$. collo-cygni generally are able to infect a broad range of host species or if host specialization (e.g., to barley) occurs within local populations. Additionally, there are limited insights about $R$. collo-cygni genetic diversity and spatial structuring of populations. An amplified fragment-length polymorphism (AFLP) study investigating the population structure of samples from barley in the Czech Republic, Germany, Slovakia, and Switzerland showed variability between the samples and rejected the hypothesis of random mating in the field, but it suggested that mixed reproduction is likely (Leisova-Svobodova et al. 2012).

No teleomorph of $R$. collo-cygni has been described. However, data from two studies, one using AFLPs and another using microsatellites (Hjortshøj et al. 2013; Piotrowska et al. 2016), hint that sexual reproduction might take place in the field. Moreover, both studies show considerable variation between their two sample locations (Scotland and Denmark or Scotland and Czech Republic, respectively), but they do not reveal clear substructures within the populations (e.g., within one country).

Here, we use the our urug2 reference genome for a population genetics approach to get a deeper insight in the biology of $R$. collocygni and better understand the lifecycle. We compare two previously published genome sequences and 17 additional sequenced isolates from a global collection from different hosts. Our aims are (i) to infer the pathogen's population structure and its global diversity, (ii) to detect breakage points on the genome and assess whether $R$. collo-cygni is indeed undergoing sexual reproduction in the field, and (iii) to infer the past demographic history (i.e., changes in population sizes) of the pathogen.

\section{MATERIALS AND METHODS}

Cultures, media, and incubation conditions. For this study, 19 different isolates were used. We used 14 isolates from different geographical locations and 5 isolates from different hosts (Table 1). Isolates were selected to include representatives of all different epidemiological zones of Europe, where the species was first described and where the first "reoccurrence" as a pathogen was reported. Moreover, Europe is the continent with the most frequent observations of strong epidemics, and thus, it is possibly the center of the emergence. For the comparison, several geographical outliers and a selection of isolates from alternative hosts were obtained.

Isolation and storage were carried out according to the method developed by Peter Frei (Agroscope Switzerland, personal communication). Spores were transferred with a needle from conidiophores of symptomatic leaves onto a petri dish with clear water agar. With the aid of sterile water, the spores were plated out over the surface of the dish and incubated at room temperature under 12-h light conditions. After $24 \mathrm{~h}$, germinating single spores were picked and transferred to a new dish with potato dextrose agar (PDA; $4 \mathrm{~g}$ of potato extract and $20 \mathrm{~g}$ of dextrose) with antibiotics (aureomycin, $25 \mathrm{mg} / \mathrm{ml}$ ). After 7 days of additional incubation at room temperatures, typical cultures were selected and transferred to dishes of 1/4-strength PDA for additional growth of single cultures. For long-term storage, mycelium was cut into small plugs and transferred into sterile screw-top tubes containing 1/4-strength potato dextrose broth (without agar). The tubes were stored at 4 to $10^{\circ} \mathrm{C}$ in the dark until further use.

DNA, library preparation, and sequencing. Mycelia from above-mentioned cultures were harvested, and finely ground mycelium was used for DNA extraction as described in detail before (supplementary methods of Stam et al. 2018); in short, mycelium was flash frozen, and DNA was extracted using a classical cetrimonium bromide (CTAB) buffer and phenol/chloroform extraction. All samples were treated with ribonuclease during the first extraction step, and the DNA was washed and precipitated twice using $70 \%$ ethanol before resuspending in Tris buffer.

A sequencing library was made using the TruSeq DNA HT prep Kit with paired end chemistry $(2 \times 100 \mathrm{bp}$; insert size, $180 \mathrm{bp})$ following the manufacturer's protocols. Sequencing was performed by NGS@ TUM using two lanes on an Illumina HiSeq 2500 sequencer.

Single-nucleotide polymorphism calling and summary statistics. Sequence data were quality checked and trimmed using

TABLE 1. Overview of the sequenced samples, including information on origin (geographic coordinates: latitude and longitude in decimal degrees), as well as obtained read depth and number of polymorphisms per sample after stringent filtering (low read quality [qual < 35]; low mapping quality [mq < 40]; Fischer strand difference [fs > 60]; low read depth [depth $<10$ ]; and clustering of polymorphisms [cluster window 10; cluster size 3]) ${ }^{\mathrm{a}}$

\begin{tabular}{|c|c|c|c|c|c|c|}
\hline Strain code & Strain name & Origin & Host & Coordinates & $\begin{array}{c}\text { Average read } \\
\text { depth }\end{array}$ & $\begin{array}{l}\text { SNPs after } \\
\text { filtering }\end{array}$ \\
\hline 100413 & Switzerland & Switzerland & Barley & $47.609577,9.146538$ & 89 & 30,800 \\
\hline 100414 & Czech Republic & Czech Republic & Barley & $49.237393,17.538407$ & 88 & 29,005 \\
\hline 100415 & Germany & Germany & Barley & $48.400360,11.714086$ & 71 & 29,387 \\
\hline 100416 & Poland & Poland & Barley & $53.282751,17.362838$ & 114 & 25,128 \\
\hline 100417 & Russia & Russia & Barley & $45.090536,38.912033$ & 97 & 26,450 \\
\hline 100418 & Hungary & Hungary & Barley & $47.1625,19.5033$ & 109 & 27,967 \\
\hline 100419 & New Zealand & New Zealand & Barley & $-43.33000,172.28000$ & 108 & 33,683 \\
\hline 100420 & Argentina & Argentina & Barley & $-33.891281,-60.574599$ & 98 & 42,167 \\
\hline 100421 & Scotland & Scotland & Barley & $55.86090,3.20640$ & 97 & 25,702 \\
\hline 100422 & Denmark & Denmark & Barley & $55.316667,11.400000$ & 93 & 26,770 \\
\hline 100423 & France & France & Barley & $47.87950,2.773600$ & 87 & 26,095 \\
\hline 100424 & England & England & Barley & $50.864600,0.255200$ & 95 & 23,510 \\
\hline 100425 & Norway & Norway & Barley & $63.430500,10.395100$ & 97 & 44,390 \\
\hline 100426 & Wheat & Germany & Winter wheat & $48.400360,11.714086$ & 102 & 23,233 \\
\hline 100427 & Tritordeum & Germany & Tritordeum & $48.400360,11.714086$ & 102 & 26,582 \\
\hline 100428 & Oat & Germany & Oat & $48.400360,11.714086$ & 97 & 28,866 \\
\hline 100429 & Agropyron & Switzerland & Agropyron repens & $46.398888,6.233293$ & 94 & 28,323 \\
\hline 100430 & Poa & Switzerland & Роа аппиа & $46.398888,6.233293$ & 88 & 29,665 \\
\hline Urug2 & Uruguay & Uruguay & Barley & $-34.334326,-57.691932$ & 190 & 57 \\
\hline
\end{tabular}

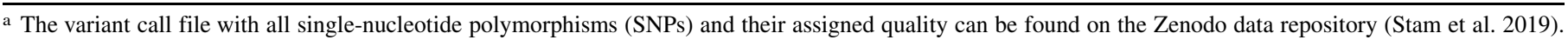


trimmomatic (recommended settings) (Bolger et al. 2014). All samples were mapped to our reference genome (Stam et al. 2018) using the Burrows-Wheeler alignment tool (Li and Durbin 2009). The duplicated reads were removed, and single-nucleotide polymorphism (SNP) calling was performed using GATKs HaplotypeCaller (McKenna et al. 2010) with ploidy set to one. SNPs were subsequently filtered using GATKs recommended filtering criteria for read and mapping quality. SNPs were filtered out when they had low quality (qual $<35$ ), low mapping quality $(\mathrm{mq}<40$ ), and high Fisher strand difference (fs $>60$ ). Also, regions with very low overall reads mapped (depth $<10$ ) were removed. In addition, we removed SNP around indels and SNPs occurring in clusters (more than three SNPs within a 10-bp window). The variant call file (vcf) with all SNPs and their assigned quality can be found in the Zenodo data repository (Stam et al. 2019). Vcftools (Danecek et al. 2011) was used to extract the summary statistics, calculate the transition to transversion ratio, and convert the vcfs to PLINK phenotype files that can be used for additional analysis. Data for the site frequency spectrum were directly extracted from the vcfs and visualized in $\mathrm{R}$ (R Core Team) using the package ggplot2 (Wickham 2009). Tajima's $D$ and nucleotide diversity $(\pi)$ were calculated over the genome using the R package PopGenome (Pfeifer et al. 2014) and reported per gene as average per site value.

Population structure and phylogeny. To assess the population structure, we used the LEA package (Frichot and François 2015) in R. We analyzed basic population structure by drawing a principal component analysis (PCA) for all data using the $\mathrm{pca}$ () function. We calculated the minimal cross-entropy (mce) for the dataset; mce is lowest when using low $K$ values, and thus, we choose $K=2$ to 4 for additional analysis. We performed 10 runs with $\alpha$ set to 10 and 100 . Both analyses were run for both all SNPs as well as SNPs in coding regions only. Differences between all of these runs were minimal; thus, only results for a single run (with $\alpha=10$, SNPs in coding regions only) were plotted. To construct a population phylogeny, we extracted all 162,045 SNPs as alternative reference genomes using GATK and drew an unrooted neighborhood joining tree using PhyML in Seaview (using the general timereversible [GTR] model and allowing nearest neighbor interchange [NNI] with BioNJ and 1,000 bootstraps) (Gouy et al. 2010; Guindon and Gascuel 2003).

Linkage disequilibrium analysis using LDHelmet. We calculated the recombination rate in $\rho$ per kilobase using the software LDHelmet (Chan et al. 2012). LDHelmet requires long scaffolds to calculate breakage points. We performed the analysis on scaffolds 1 to 12 , because the others are shorter than $1 \mathrm{Mb}$. The software was run using the guidelines provided by the authors. We used a window size of 50 and recommended parameters for $\rho$ (recombination) search space. We specified a burn in of 100,000 iterations and 1,000,000 true iterations. Block penalty $(-\mathrm{b})$ was set to 50 . The results for the upper and lower 2.5 percentile as well as the median were extracted from the binary results file and imported into $\mathrm{R}$ for visualization. The histogram with $\rho$ values per bin was plotted using the R package ggplot2. Linkage disequilibrium graphs were plotted using the base plot function. These analyses were done once for all samples and once for a subset of the samples excluding the three outliers as identified by the phylogeny (Argentina, Norway, and New Zealand).

Estimation of recombination events with ClonalFrameML. The genomic sequences for scaffolds 1 and 2 were extracted for each strain. A phylogenetic tree was constructed using PhyML in Seaview (GTR, NNI, BioNJ, and 100 bootstraps) (Gouy et al. 2010; Guindon and Gascuel 2003). Next, we used ClonalFrameML (Didelot and Wilson 2015) to estimate recombination events. As prior for the transition to transversion ratio, we used the calculated rate of 2.3. Other priors were set as follows: $1 / \rho$ was taken from our LDHelmet analysis, the minimum length for each recombination was set to $10 \mathrm{~kb}, \mathrm{v}$ (mean divergence of imported DNA) was set to 0.1 as recommended in the manual, and simulations were run three times, each time with different genomic mutation rates of $1 \times 10^{-7}, 1 \times 10^{-7}, 1 \times 10^{-8}$, and $1 \times 10^{-9}$. As recommended in the manual, we set the allowed standard deviation for the estimations such that we allowed several orders of magnitude variation in our estimation. The number of recombination events was extracted from the result file and visualized as events per megabase using ggplot 2 in $\mathrm{R}$.

Historic effective population size estimation. For the estimation of the historic effective population size (Ne), we used the software PopSizeABC (Boitard et al. 2016). PopSizeABC works best with long scaffolds; therefore, we calculated summary statistics for the first 12 scaffolds (each $>1-$ Mb long). We performed 200,000 simulations with each of 50 independent fragments of 2 $\mathrm{Mb}$. Parameters for $\rho$ were derived from the LDHelmet results, with a minimum cutoff at $1 \times 10^{-8}$ and a maximum at $1 \times 10^{-7}$ set as priors. Minimum allele frequency was set at 2, and the maximum Ne was set at 1,000,000. Summary statistics were extracted using the same parameters, with the tolerance, as recommended, set to 0.0005 .

The ABC method was repeated for generation times of $0.25,0.5$, 1,3 , and 5 years. The graphs were plotted in $\mathrm{R}$ using a modification of the script provided by PopSizeABC to allow for visualization of 100 individual runs.

Data availability. Sequencing data are available from the European Nucleotide Archive (accession no. PRJEB12938; https:// www.ebi.ac.uk/ena/data/search?query=PRJEB12938). The vcf is available in Zenodo at https://zenodo.org/record/3256422 (Stam et al. 2019).

\section{RESULTS}

Sequencing $R$. collo-cygni strains. All reads from the sequenced isolates were mapped to our reference genome. Average read depth ranged from $64 \times$ to $113 \times$ after deduplication (Table 1 ). SNPs were called using GATK (McKenna et al. 2010). In total, we obtained 319,477 SNPs. To verify robust SNP call, we used stringent settings for mapping and SNP quality; 157,433 SNPs were filtered out, 128,943 of which because of low mapping quality. We kept 162,045 high-confidence SNPs between all samples, corresponding to approximately one SNP in every 200 bases. The file with all SNP calls and their quality is available online (Stam et al. 2019). The number of SNPs called in each of the individual samples ranged from 23,233 to 44,390 , with a mean of 29,317 SNPs (Table 1). Thus, on average, each sample has one SNP every $\sim 1,090$ bp compared with the reference. In the whole dataset, 79,949 SNPs occurred within coding regions. We calculated a transition to transversion ratio of 2.36 for the whole genome and 2.31 for the coding sites, suggesting no anomalies in the SNP calling. Of all SNPs in the coding regions, $56 \%$ occur as singletons within the sample set (Supplementary Fig. S1). Average nucleotide diversity per site $(\pi)$ over all coding sites was $4.7 \times 10^{-4}$, and Tajima's $D$ was -1.186 . When looking at per gene statistics, median value of $\pi$ per site is $4.7 \times 10^{-4}$, and Tajima's $D$ was -1.1630 . Such overall low diversity, high singleton rate, and low Tajima's $D$ are indicative of a population expansion, strong selective constraints, or pervasive positive selection (selective sweeps).

$\boldsymbol{R}$. collo-cygni population structure and phylogeny. We further used several approaches to assess the population structure of $R$. collo-cygni. We first reduced the complexity of the data and constructed a PCA for the SNPs using LEA, an R package used to calculate admixture (Frichot and François 2015). Supplementary Figure S2 shows that the first two components explain 17\% (12 and $5)$ of the variation. When we drew the two first components, most isolates grouped very closely together in one cluster. Only three outliers (Norway, Argentina, and New Zealand) could be observed. Similar patterns appeared when using the coding regions of the genome only as well as in the whole genome. Next, we estimated the population structure from the SNP data. The previous PCA results 
and minimum entropy analysis (Supplementary Fig. S3) suggested the optimal cluster $K$ value to be between one and three. We ran LEA, for $K$ values $=2$ to 4 , in 10 separate runs. As expected from the PCA, Figure 1A shows that, with these $K$ values, two or three isolates could be marked as outliers, being the isolates from Norway, Argentina, and possibly, New Zealand. All other samples grouped together with $K=3$ and showed a transient pattern for $K=4$. Segregation of isolates could not be seen on a geographical basis or based on the host of origin.

To confirm these findings, we extracted all high-confidence polymorphic sites from the reconstructed genome sequence for each of the isolates. The resulting alignment was used to construct a phylogeny using PhyML (NNI, 1,000 bootstraps). Figure 1B shows that isolates from Norway and Argentina were indeed the two main outliers followed by New Zealand. Using the same shading in both panels revealed very similar internal structures. The low bootstrap values at some of the internal branches of the phylogeny indicate that no clear subgroups can be defined within the inner branches of

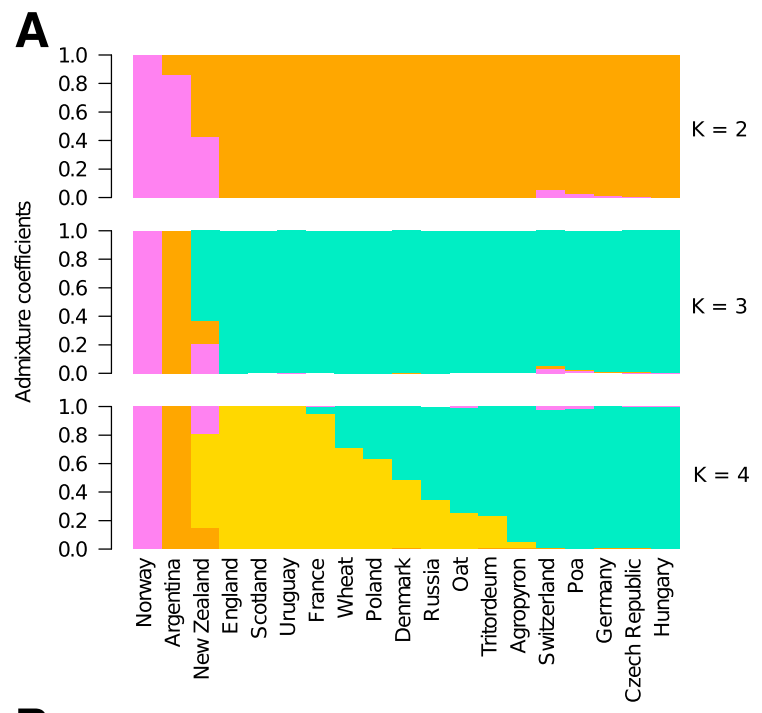

B

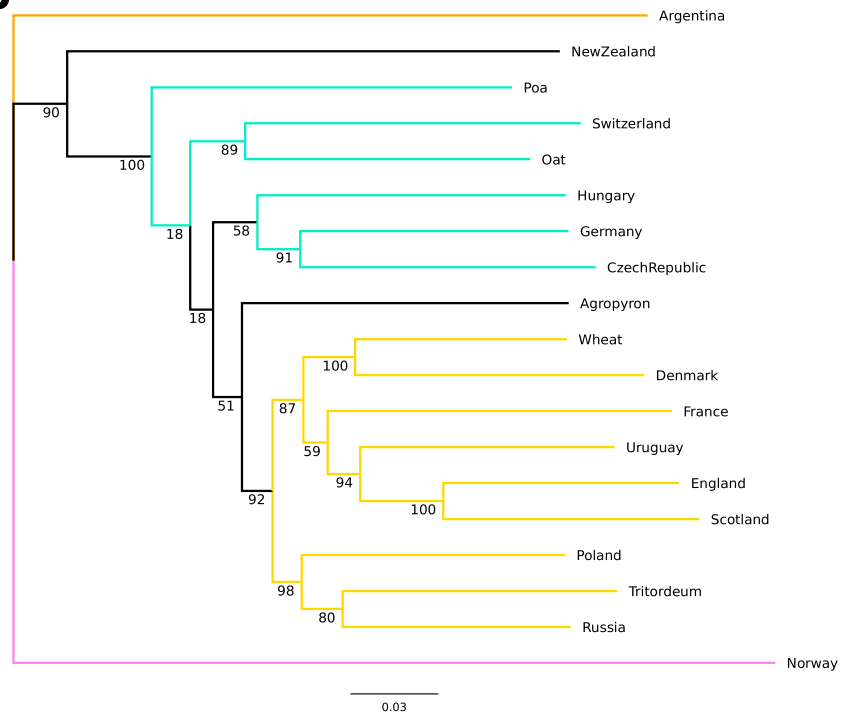

Fig. 1. Global Ramularia collo-cygni substructure. A, Admixture plots for $K+$ 2 to 4 . Each vertical bar ( $x$ axis) represents a different isolate. Gray shading illustrates the different assigned genotypes or admixture thereof when a bar has more shades on the $y$ axis. $\mathbf{B}$, Phylogenetic reconstruction based on all single-nucleotide polymorphisms identified between the 19 sequenced $R$. collo-cygni isolates of our study. The phylogeny was made using PhyML (general time-reversible model and nearest neighbor interchange; BioNJ; 1,000 bootstraps). Bootstrap values are indicated on the individual branches. Branches are shaded as in $\mathbf{A}$. the tree. Thus, except for three outliers, we did not find a clear population structure for $R$. collo-cygni in our dataset.

Analyses of sexual recombination. Next, we investigated whether there are signatures of sexual recombination within the species. We used LDHelmet (Chan et al. 2012) to estimate the linkage disequilibrium within the species on the longest scaffolds of our assembly (those $>1-\mathrm{Mb}$ long). Figure 2 and Supplementary Figure S4 show that, when analyzing all 19 isolates, rare but very clear changes in linkage disequilibrium events could be observed. We calculated $\rho$ values across the genome that were mostly $<10^{-6}$ / $\mathrm{kbp}$. However, in small windows, these values ranged up to eight (Supplementary Fig. S5). When we repeated the LDHelmet analysis without the three aforementioned outliers, these peaks in $\rho$ became lower, but they remained present (Fig. 2).

To confirm our findings, we used ClonalFrameML (Didelot and Wilson 2015) to analyze recombination on the first two scaffolds within our set of $R$. collo-cygni isolates. ClonalFrameML uses genome alignments to screen for breakpoints that are indicative of recombination events. We took the priors $1 / \rho$ and transition to transversion ratio from our analyses above. The expected mutation rate of the genome of $R$. collo-cygni is unknown, but genomic mutation rates in related eukaryotic microorganisms generally range from $1 \times 10^{-6}$ to $1 \times 10^{-9}$ per $1 \mathrm{bp}$, with $1 \times 10^{-7}$ to $1 \times 10^{-8}$ the most likely rate for multicellular fungi, like $R$. collo-cygni (Drake et al. 1998). For each of the 19 sampled strains, we calculated the expected number of recombination events. The number of events varied per strain. In all cases, we saw strong evidence for low rates of recombination, with median rates ranging from six to four recombination events per $1 \mathrm{Mb}$ on both scaffolds 1 and 2 (Fig. 3). This indicates that sexual reproduction indeed happened to a certain extent through the whole sample set.

Estimation of the Ne. All our estimates of Ne revealed one main fluctuation (Supplementary Fig. S6): a historically large Ne of $>100,000$, with a gradual decline over time, where Ne dropped as low as 100. After that, Ne gradually increased again, and it has remained relatively stable in the three latest iterations of the Ne size estimations. With a generation time set to 1 year (a realistic scenario
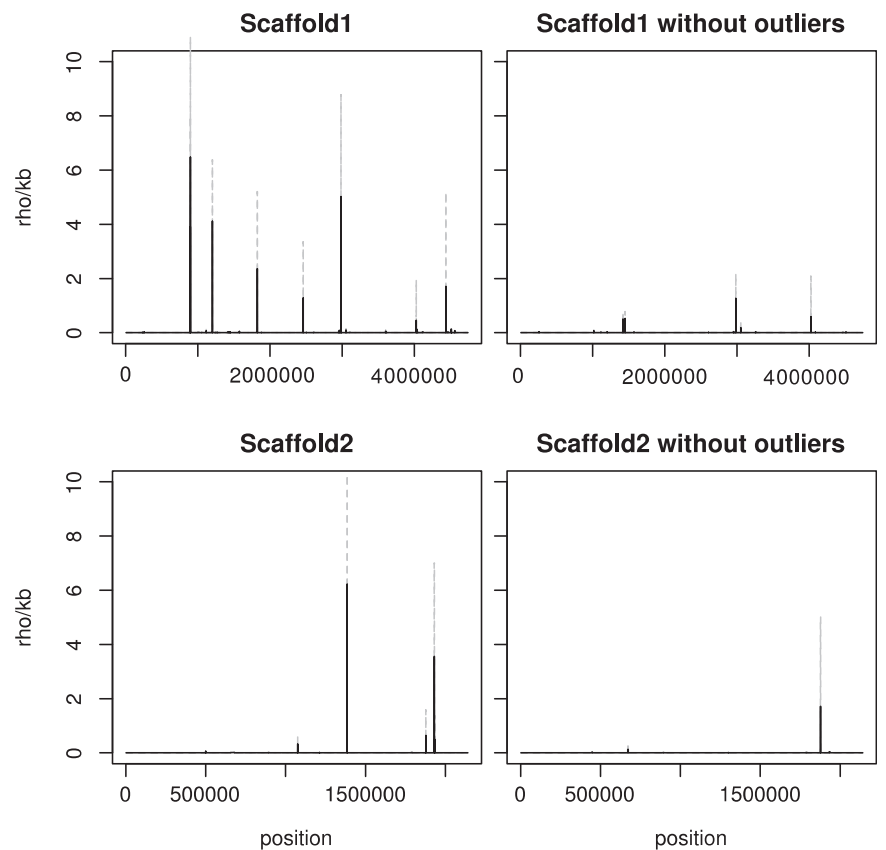

Scaffold2 without outliers

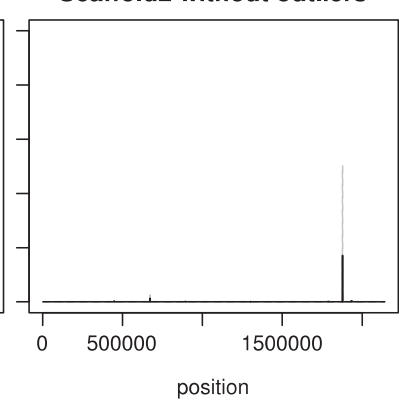

Fig. 2. Recombination analyses in Ramularia collo-cygni using LDHelmet. Recombination in $\rho$ per kilobase ( $y$ axis) was calculated per position on the scaffold for the 12 longest scaffolds $(>1,000,000 \mathrm{bp}$ ). Scaffolds 1 and 2 are shown. Left panels show the results for all samples, and right panels show the results excluding the three outlier samples. The $x$ axis shows the scaffold length in base pairs. 
seeing the endophytic growth and symptom appearance late in each season), we estimated that $R$. collo-cygni saw a peak in population growth about 10,000 years ago; then, it underwent a bottleneck, with the lowest Ne between 300 and 1,500 years ago. Since then, the $\mathrm{Ne}$ has increased 100-fold to about 100,000 (Fig. 4).

\section{DISCUSSION}

R. collo-cygni was discovered as a saprophyte of grasses in the 1890s (Cavara 1893). Since the 1980s, it has attracted increasing attention as the causal agent for RLS. To obtain a better understanding of the population structure and the role of sexual reproduction in the current worldwide emergence of $R$. collo-cygni, we analyzed $19 R$. collo-cygni strains. We reveal a relatively low genetic diversity. We found one SNP per 200 bases in 19 samples compared with, for example, one SNP per 20 bases in 106 global Z. tritici samples (Hartmann et al. 2017). We also observe a very similar transition to transversion ratio on coding regions and the whole genome (2.31 and 2.37, respectively). These findings are a first indication of a possible bottleneck and expansion of the species. There is very little evidence for geographical population substructure. With the exception of three possible outlier samples, all of our samples form one genetic cluster. In a previous study, populations from the Czech Republic and Scotland were found to form two moderately diverged populations that could already be part of the same genetic cluster (Piotrowska et al. 2016). With our additional sampling, we show that isolates from these populations belong within the same large main cluster that covers a large geographic area. The main cluster can be divided in two putative genomic groups, but these do not show a strong geographical correlation. These results are partly surprising, because other cereal pathogens, such as the primarily windborne wheat yellow rust fungus Puccinia striiformis f. sp. tritici (Ali et al. 2014) and also, Fusarium graminearum (another partially seedborne cereal pathogen) (O'Donnell et al. 2000), do show geographically distinct lineages.

One explanation for lack of geographical clustering of the sample could be long-distance dispersal of the pathogen. As discussed, wind dispersal could be one means by which $R$. collo-cygni spores travel around the globe (Stabentheiner et al. 2009; Walters et al. 2008). Such a mode of dispersal is heavily reliant on stochastic processes (Brown and Hovmøller 2002), but that would only in part explain the lack of geographical clustering. In a wind dispersal scenario, geographically close areas are often most likely to be genetically more similar to one another. A more likely explanation for the lack of geographical structure could be seed transmission originating from contaminated seed shipment. Barley seeds are often shipped around the globe, and the effect could be amplified by maintenance of contaminated barley in winter nurseries. Such seed transmission of $R$. collo-cygni has been suggested before (Havis et al. 2014) and would be in line with observations made for cereal pathogens, like Magnaporthe oryzae (Islam et al. 2016) and Rynchosporium commune (Linde et al. 2009). It also fits that $R$. collo-cygni is regularly detected inside the grain (Matusinsky et al. 2011). Interestingly, the most closely related sister species to R. collo-cygni, Z. tritici, is rarely detected in seed (Suffert et al. 2011).

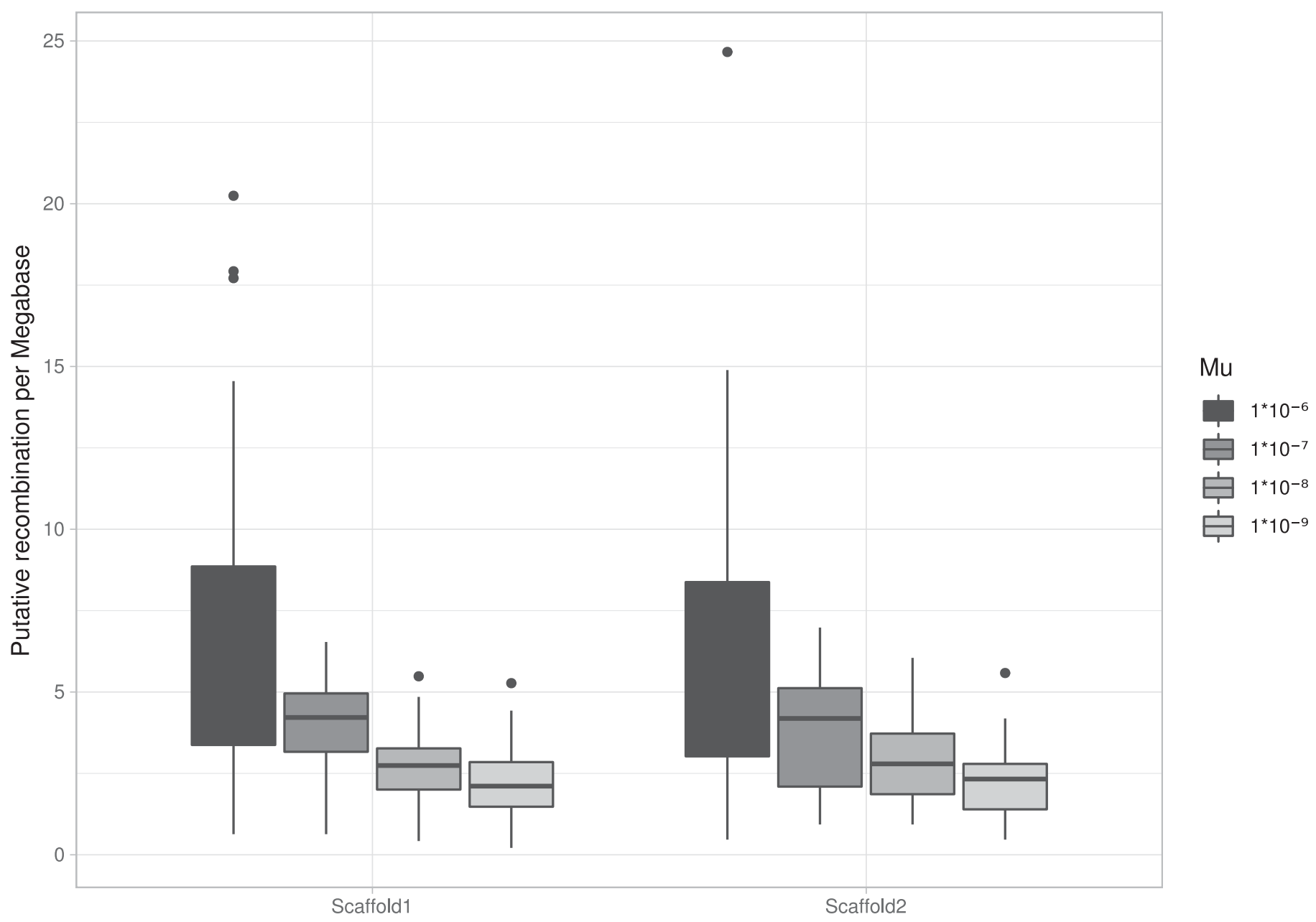

Fig. 3. Recombination analyses using ClonalFrameML. The number of expected recombinations per megabase per isolate as calculated by ClonalFrameML ( $y$ axis) is plotted for scaffolds 1 and 2 ( $x$ axis). The analyses were done with four different assumed genomic mutation rates $(\mu)$ ranging from $1 \times 10^{-6}\left(\right.$ black) to $1 \times 10^{-9}$ (light gray). Two are estimated for different genomic mutation rates ( $x$ axis). 
Our analyses also show no sign of a clear host specialization, because none of the outliers stem from an alternative host. These results are in contrast with many other fungi. For example, the rice blast fungus $M$. oryzae is pervasive on rice, but specific strains can also be found on many other monocot hosts (Gladieux et al. 2018; Yoshida et al. 2016). Z. tritici contains a small set of genes that can be related to host preference (Poppe et al. 2015), and even the broadhost range, opportunistic pathogen Botrytis cinerea shows signs of host adaptation (Leyronas et al. 2015). It will be crucial to identify other factors in the biology of $R$. collo-cygni that determine its generalist properties. This is particularly relevant, because other wild gramineous hosts can be an alternative source of inoculum. This can result in more than one overwintering host being available that can provide a "green bridge" or "seed bridge" for $R$. collo-cygni to survive winter. Yet, it has to be noted that analyses from spore traps report very little spore movement until later in the season (Havis et al. 2015).

The lack of host adaptation on the fungal side could be further evidence for $R$. collo-cygni being a general endophyte of grasses. Pathogenesis would then derive from susceptibility factors from the host genetic side, likely triggered or at least enhanced by environmental factors. In Piriformospora indica, metabolic cues from its hosts result in different levels of colonization by directly affecting expression levels of genes related to specific lifestyles
(Lahrmann et al. 2013). Similar specific interactions are described for symbiosis (Rodriguez and Redman 2008). In fact, early infection with $R$. collo-cygni can have beneficial effects on yield, suggesting that the host-parasite interaction switches from mutualistic via endophytic to parasitic (Newton et al. 2010). An early mutualistic interaction could also explain the lack of major resistance genes against $R$. collo-cygni in barley. The absence of epidemics in the related genus Gramineae and the high diversity in barley germplasm give hope that host susceptibility factors, quantitative resistance loci, or even resistance genes can be identified for future control of RLS. However, an integrated genome-wide analysis of the host and pathogen transcriptome landscape after inoculation of a susceptible barley cultivar displayed molecular signatures reminiscent of a pathogenic fungus, triggering classical defense response pathways to necrotrophic pathogens, like ethylene and jasmonic acid signaling, in the susceptible host (Sjokvist et al. 2019). This also suggests that $R$. collo-cygni, at least partially, suppressed host defense responses in the later symptomatic stages of infection, at least under artificial inoculation conditions. Understanding the fungal virulence factors during leaf infection in various barley cultivars will thus be important for the prevention of RLS disease.

Rapid expansion of a plant pathogen can arise from beneficial recombination events during sexual reproduction followed by

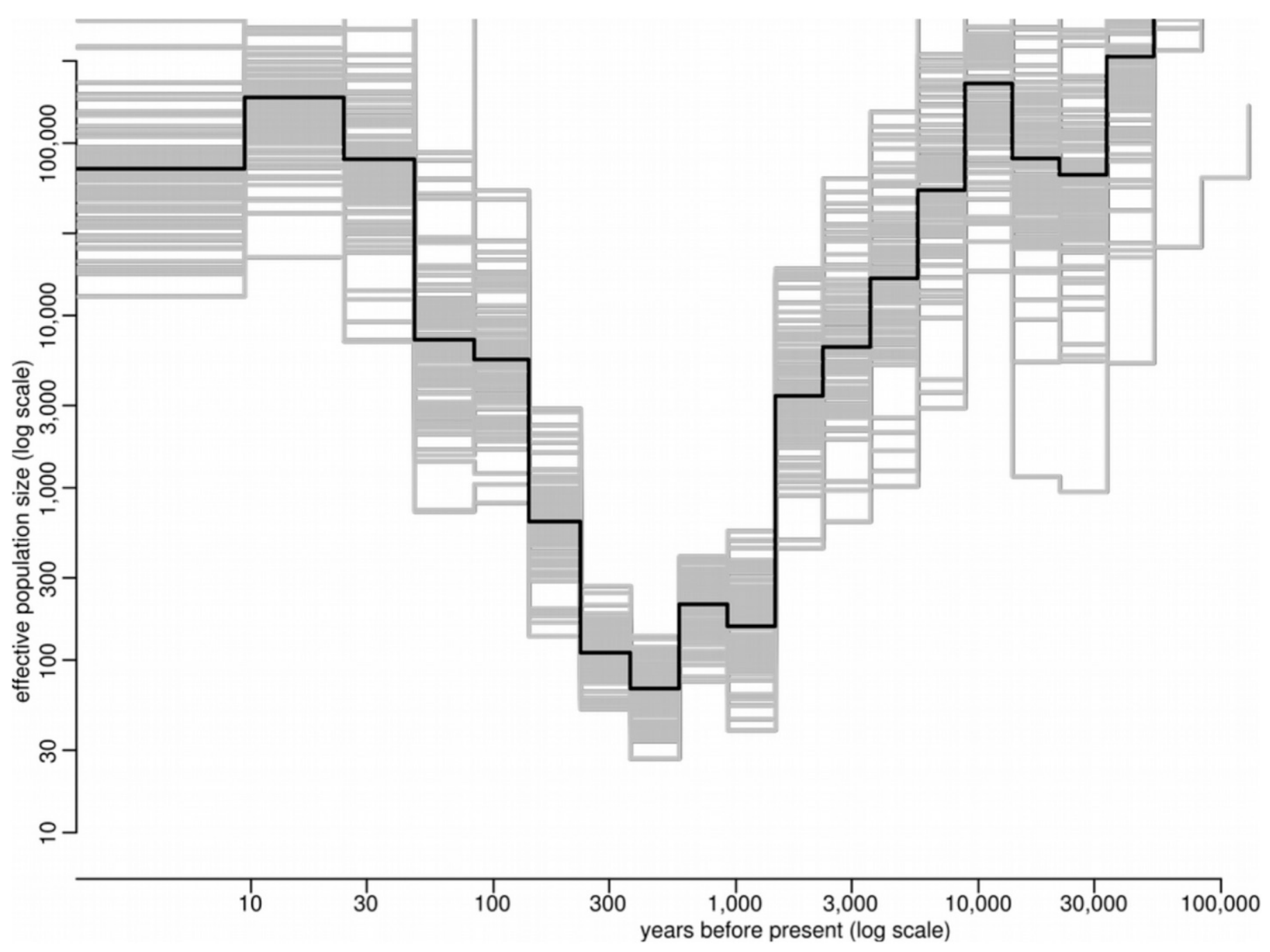

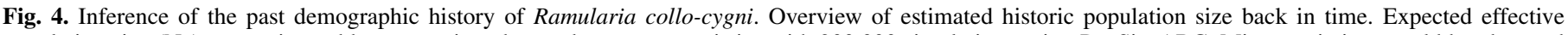

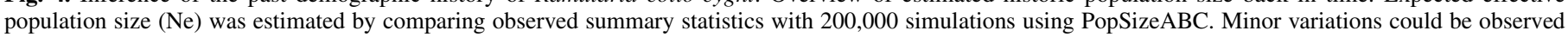

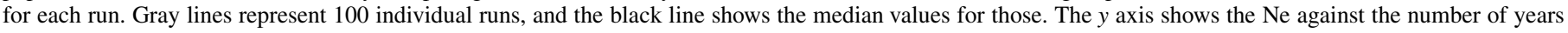
(or generations) ago ( $x$ axis). 
selection on susceptible hosts. Using linkage disequilibrium scans and recombination analyses, we show that indeed there is evidence of sexual recombination within the collection of isolates that we inspected. This supports previous hypotheses (Hjortshøj et al. 2013; Piotrowska et al. 2016). Interestingly, the linkage disequilibrium scan signal strength for recombination is lower in the main subpopulation (or main isolate cluster) that excludes Argentina, Norway, and New Zealand. This could indicate that the expansion of $R$. collo-cygni is possibly partly attributed to clonal propagation with episodic sexual recombination. The combination of high clonal reproduction and global transfer via seed was probably favored by modern intensification of cereal production and global cereal food, fodder and seed trade, and trafficking.

When calculating the effective population size over time, we see an increase shortly after domestication of barley (10,000 years ago) followed by a gradual decrease. The past 300 years show a gradual increase again. This coincides with the intensification and switch to monocultures of barley, suggesting that $R$. collo-cygni expanded over time with the increased availability of susceptible hosts (Hjortshøj et al. 2013; Piotrowska et al. 2016). We speculate that this could have happened after recombination of strains that occurred as endophytes in wild grasses; this is a scenario similar to tan spot on wheat, which was frequently found in wild grasses and transferred to wheat only in the 1940s (Friesen et al. 2006). Yet, additional sampling, particularly from wild grasses, would be required to confirm this scenario.

With its relatively large effective population size compared with Z. tritici (Stukenbrock et al. 2011), likely mixed reproduction, and global gene flow, $R$. collo-cygni meets the criteria to be classified as a high-risk pathogen (McDonald and Linde 2002). No major R genes have been identified against $R$. collo-cygni in barley, and the characteristics described above suggest that, even if $\mathrm{R}$ genes were to be identified, they are unlikely to provide durable resistance, even when introduced as stacks or pyramids (Stam and McDonald 2018). Similarly, fungicide resistance can evolve rapidly and spread throughout the global populations owing to strong positive selection combined with recombination and extensive gene flow. Thus, the best way to diminish the current epidemic would be to drastically reduce the effective population size. This could be achieved by measurements to reduce overwintering capacity and transfer of infected seed or more diverse breeding approaches, including identification of host factors that result in quantitative resistances or manipulation of host susceptibility factors.

\section{ACKNOWLEDGMENTS}

We thank Christine Wurmser (Chair of Animal Breeding, Technical University of Munich) for support with sequencing and Carolin Hutter and Regina Dittebrand for technical assistance. We acknowledge the community of "Ramulariosi" for providing samples and isolates, in particular Peter Frei, Pavel Matusinsky, and Neil Havis.

\section{LITERATURE CITED}

Ali, S., Gladieux, P., Leconte, M., Gautier, A., Justesen, A. F., Hovmøller, M. S., Enjalbert, J., and de Vallavieille-Pope, C. 2014. Origin, migration routes and worldwide population genetic structure of the wheat yellow rust pathogen Puccinia striiformis f.sp. tritici. PLoS Pathog 10: e1003903.

Boitard, S., Rodríguez, W., Jay, F., Mona, S., and Austerlitz, F. 2016. Inferring population size history from large samples of genome-wide molecular data - an approximate Bayesian computation approach. PLoS Genet. 12: e1005877.

Bolger, A. M., Lohse, M., and Usadel, B. 2014. Trimmomatic: A flexible trimmer for Illumina sequence data. Bioinformatics 30:2114-2120.

Brown, J. K. M., and Hovmøller, M. S. 2002. Aerial dispersal of pathogens on the global and continental scales and its impact on plant disease. Science 297:537-541.

Cavara, F. 1893. Über einige parasitische Pilze auf dem Getreide. Z. Fur Pflanzenkrankh. 3:16-26.
Chan, A. H., Jenkins, P. A., and Song, Y. S. 2012. Genome-wide fine-scale recombination rate variation in Drosophila melanogaster. PLoS Genet. 8: e1003090.

Danecek, P., Auton, A., Abecasis, G., Albers, C. A., Banks, E., DePristo, M. A., Handsaker, R. E., Lunter, G., Marth, G. T., Sherry, S. T., McVean, G., Durbin, R., and 1000 Genomes Project Analysis Group. 2011. The variant call format and VCFtools. Bioinformatics 27:2156-2158.

Didelot, X., and Wilson, D. J. 2015. ClonalFrameML: Efficient inference of recombination in whole bacterial genomes. PLOS Comput. Biol. 11: e1004041.

Drake, J. W., Charlesworth, B., Charlesworth, D., and Crow, J. F. 1998. Rates of spontaneous mutation. Genetics 148:1667-1686.

Frichot, E., and François, O. 2015. LEA: An R package for landscape and ecological association studies. Methods Ecol. Evol. 6:925-929.

Friesen, T. L., Stukenbrock, E. H., Liu, Z., Meinhardt, S., Ling, H., Faris, J. D., Rasmussen, J. B., Solomon, P. S., McDonald, B. A., and Oliver, R. P. 2006. Emergence of a new disease as a result of interspecific virulence gene transfer. Nat. Genet. 38:953-956.

Gladieux, P., Condon, B., Ravel, S., Soanes, D., Maciel, J. L. N., Nhani, A., Jr., Chen, L., Terauchi, R., Lebrun, M.-C., Tharreau, D., Mitchell, T., Pedley, K. F., Valent, B., Talbot, N. J., Farman, M., and Fournier, E. 2018. Gene flow between divergent cereal- and grass-specific lineages of the rice blast fungus Magnaporthe oryzae. MBio. 9:e01219-17.

Gouy, M., Guindon, S., and Gascuel, O. 2010. SeaView version 4: A multiplatform graphical user interface for sequence alignment and phylogenetic tree building. Mol. Biol. Evol. 27:221-224.

Guindon, S., and Gascuel, O. 2003. A simple, fast, and accurate algorithm to estimate large phylogenies by maximum likelihood. Syst. Biol. 52:696-704.

Hartmann, F. E., Sánchez-Vallet, A., McDonald, B. A., and Croll, D. 2017. A fungal wheat pathogen evolved host specialization by extensive chromosomal rearrangements. ISME J. 11:1189-1204.

Havis, N. D., Brown, J. K., Clemente, G., Frei, P., Jedryczka, M., Kaczmarek, J., Kaczmarek, M., Matusinsky, P., McGrann, G. R. D., Pereyra, S., Piotrowska, M., Sghyer, H., Tellier, A., and Hess, M. 2015. Ramularia collo-cygni-an emerging pathogen of barley crops. Phytopathology 105: 895-904.

Havis, N. D., Nyman, M., and Oxley, S. J. P. 2014. Evidence for seed transmission and symptomless growth of Ramularia collo-cygni in barley (Hordeum vulgare). Plant Pathol. 63:929-936.

Hjortshøj, R. L., Ravnshøj, A. R., Nyman, M., Orabi, J., Backes, G., Pinnschmidt, H., Havis, N., Stougaard, J., and Stukenbrock, E. H. 2013. High levels of genetic and genotypic diversity in field populations of the barley pathogen Ramularia collo-cygni. Eur. J. Plant Pathol. 136:51-60.

Hofer, K., Linkmeyer, A., Textor, K., Hückelhoven, R., and Hess, M. 2015. MILDEW LOCUS O mutation does not affect resistance to grain infections with Fusarium spp. and Ramularia collo-cygni. Phytopathology 105: 1214-1219.

Islam, M. T., Croll, D., Gladieux, P., Soanes, D. M., Persoons, A., Bhattacharjee, P., Hossain, S., Gupta, D. R., Rahman, M., Mahboob, M. G., Cook, N., Salam, M. U., Surovy, M. Z., Bueno Sancho, V., Nunes Maciel, J. L., Nhani-Júnior, A., Castroagudín, V. L., de Assis Reges, J. T., Ceresini, P. C., Ravel, S., Kellner, R., Fournier, E., Tharreau, D., Lebrun, M.-H., McDonald, B. A., Stitt, T., Swan, D., Talbot, N. J., Saunders, D. G. O., Win, J., and Kamoun, S. 2016. Emergence of wheat blast in Bangladesh was caused by a South American lineage of Magnaporthe oryzae. BMC Biol. $14: 84$.

Kaczmarek, M., Piotrowska, M. J., Fountaine, J. M., Gorniak, K., McGrann, G. R. D., Armstrong, A., Wright, K. M., Newton, A. C., and Havis, N. D. 2017. Infection strategy of Ramularia collo-cygni and development of ramularia leaf spot on barley and alternative graminaceous hosts. Plant Pathol. 66:45-55.

Lahrmann, U., Ding, Y., Banhara, A., Rath, M., Hajirezaei, M. R., Döhlemann, S., von Wirén, N., Parniske, M., and Zuccaro, A. 2013. Host-related metabolic cues affect colonization strategies of a root endophyte. Proc. Natl. Acad. Sci. 110:13965-13970.

Leisova-Svobodova, L., Matusinsky, P., and Kucera, L. 2012. Variability of the Ramularia collo-cygni population in central Europe. J. Phytopathol. 160: 701-709.

Leyronas, C., Bryone, F., Duffaud, M., Troulet, C., and Nicot, P. C. 2015. Assessing host specialization of Botrytis cinerea on lettuce and tomato by genotypic and phenotypic characterization. Plant Pathol. 64:119-127.

Li, H., and Durbin, R. 2009. Fast and accurate short read alignment with Burrows-Wheeler transform. Bioinformatics 25:1754-1760.

Linde, C. C., Zala, M., and McDonald, B. A. 2009. Molecular evidence for recent founder populations and human-mediated migration in the barley scald pathogen Rhynchosporium secalis. Mol. Phylogenet. Evol. 51: 454-464.

Matusinsky, P., Leisova-Svobodova, L., Gubis, J., Hudcovicova, M., Klcova, L., Gubisova, M., Mařík, P., Tvaruzek, L., and Minarikova, V. 2011. Impact 
of the seed-borne stage of Ramularia collo-cygni in barley seed. J. Plant Pathol. 93:679-689.

McDonald, B. A., and Linde, C. 2002. Pathogen population genetics, evolutionary potential, and durable resistance. Annu. Rev. Phytopathol. 40: 349-379.

McGrann, G. R., Andongabo, A., Sjökvist, E., Trivedi, U., Dussart, F., Kaczmarek, M., Mackenzie, A., Fountaine, J. M., Taylor, J. M. G., Paterson, L. J., Gorniak, K., Burnett, F., Kanyuka, K., Hammond-Kosack, K. E., Rudd, J. J., Blaxter, M., and Havis, N. D. 2016. The genome of the emerging barley pathogen Ramularia collo-cygni. BMC Genomics 17:584.

McGrann, G. R. D., Stavrinides, A., Russell, J., Corbitt, M. M., Booth, A., Chartrain, L., Thomas, W. T. B., and Brown, J. K. M. 2014. A trade off between mlo resistance to powdery mildew and increased susceptibility of barley to a newly important disease, ramularia leaf spot. J. Exp. Bot. 65: 1025-1037.

McKenna, A., Hanna, M., Banks, E., Sivachenko, A., Cibulskis, K., Kernytsky, A., Garimella, K., Altshuler, D., Gabriel, S., Daly, M. and DePristo, M. A. 2010. The Genome Analysis Toolkit: A MapReduce framework for analyzing next-generation DNA sequencing data. Genome Res. 20:1297-1303.

Newton, A. C., Fitt, B. D. L., Atkins, S. D., Walters, D. R., and Daniell, T. J. 2010. Pathogenesis, parasitism and mutualism in the trophic space of microbe-plant interactions. Trends Microbiol. 18:365-373.

O’Donnell, K., Corby-Kistler, H., Tacke, B. K., and Casper, H. H. 2000. Gene genealogies reveal global phylogeographic structure and reproductive isolation among lineages of Fusarium graminearum, the fungus causing wheat scab. Proc. Natl. Acad. Sci. USA 97:7905-7910.

Pfeifer, B., Wittelsbürger, U., Ramos-Onsins, S. E., and Lercher, M. J. 2014. PopGenome: An efficient Swiss army knife for population genomic analyses in R. Mol. Biol. Evol. 31:1929-1936.

Piotrowska, M. J., Ennos, R. A., Fountaine, J. M., Burnett, F. J., Kaczmarek, M., and Hoebe, P. N. 2016. Development and use of microsatellite markers to study diversity, reproduction and population genetic structure of the cereal pathogen Ramularia collo-cygni. Fungal Genet. Biol. 87:64-71.

Poppe, S., Dorsheimer, L., Happel, P., and Stukenbrock, E. H. 2015. Rapidly evolving genes are key players in host specialization and virulence of the fungal wheat pathogen Zymoseptoria tritici (Mycosphaerella graminicola). PLoS Pathog 11:e1005055.

Rodriguez, R., and Redman, R. 2008. More than 400 million years of evolution and some plants still can't make it on their own: Plant stress tolerance via fungal symbiosis. J. Exp. Bot. 59:1109-1114.

Salamati, S., and Reitan, L. 2006. Pages 19-35 in: Ramularia collo-cygni on Spring Barley: An Overview of Its Biology and Epidemiology. B. Koopmann, S. Oxley, A. Schützendübel, and A. von Tiedemann, eds. Georg-August-University, Göttingen, Germany.
Schützendübel, A., Stadler, M., Wallner, D., and Von Tiedemann, A. 2008. A hypothesis on physiological alterations during plant ontogenesis governing susceptibility of winter barley to ramularia leaf spot. Plant Pathol. 57: 518-526.

Sjokvist, E., Lemcke, R., Kamble, M., Turner, F., Blaxter, M., Havis, N. H. D., Lyngkjaer, M. F., and Radutoiu, S. 2019. Dissection of Ramularia leaf spot disease by Integrated analysis of barley and Ramularia collo-cygni transcriptome responses. Mol. Plant-Microbe Interact. 32:176-193.

Stabentheiner, E., Minihofer, T., and Huss, H. 2009. Infection of barley by Ramularia collo-cygni: Scanning electron microscopic investigations. Mycopathologia 168:135-143.

Stam, R., and McDonald, B. A. 2018. When resistance gene pyramids are not durable - the role of pathogen diversity. Mol. Plant Pathol. 19:521-524.

Stam, R., Münsterkötter, M., Pophaly, S. D., Fokkens, L., Sghyer, H., Güldener, U., Hückelhoven, R., and Hess, M. 2018. A new reference genome shows the one-speed genome structure of the barley pathogen Ramularia collo-cygni. Genome Biol. Evol. 10:3243-3249.

Stam, R., Sghyer, H., Tellier, A., Hess, M., and Hückelhoven, R. 2019. SNP Call Data for: The Current Epidemic of the Barley Pathogen Ramularia collo-cygni Derives from a Recent Population Expansion and Shows Global Admixture. https://zenodo.org/record/3256422

Stukenbrock, E. H., Bataillon, T., Dutheil, J. Y., Hansen, T. T., Li, R., Zala, M., McDonald, B. A., Wang, J., and Schierup, M. H. 2011. The making of a new pathogen: Insights from comparative population genomics of the domesticated wheat pathogen Mycosphaerella graminicola and its wild sister species. Genome Res. 21:2157-2166.

Suffert, F., Sache, I., and Lannou, C. 2011. Early stages of Septoria tritici blotch epidemics of winter wheat: Build-up, overseasoning, and release of primary inoculum. Plant Pathol. 60:166-177.

Walters, D. R., Havis, N. D., and Oxley, S. J. P. 2008. Ramularia collo-cygni: The biology of an emerging pathogen of barley. FEMS Microbiol. Lett. 279:1-7.

West, J. S., Townsend, J. A., Stevens, M., and Fitt, B. D. L. 2012. Comparative biology of different plant pathogens to estimate effects of climate change on crop diseases in Europe. Eur. J. Plant Pathol. 133:315-331.

Wickham, H. 2009. ggplot2: Elegant Graphics for Data Analysis. https:// ggplot2.tidyverse.org/

Yoshida, K., Saunders, D. G. O., Mitsuoka, C., Natsume, S., Kosugi, S., Saitoh, H., Inoue, Y., Chuma, I., Tosa, Y., Cano, L. M., Kamoun, S., and Terauchi, R. 2016. Host specialization of the blast fungus Magnaporthe oryzae is associated with dynamic gain and loss of genes linked to transposable elements. BMC Genomics 17:370.

Zhao, Z., Liu, H., Wang, C., and Xu, J.-R. 2013. Comparative analysis of fungal genomes reveals different plant cell wall degrading capacity in fungi. BMC Genomics 14:274. 